\title{
Controlling the polarization and vortex charge of attosecond high-harmonic beams via simultaneous spin-orbit momentum conservation
}

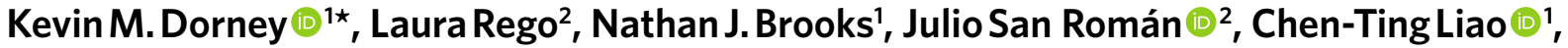 \\ Jennifer L. Ellis', Dmitriy Zusin', Christian Gentry', Quynh L. Nguyen', Justin M. Shaw3, Antonio Picón $\mathbb{1}^{2,4}$, \\ Luis Plaja², Henry C. Kapteyn', Margaret M. Murnane ${ }^{1}$ and Carlos Hernández-García ${ }^{2 \star}$
}

Optical interactions are governed by both spin and angular momentum conservation laws, which serve as a tool for controlling light-matter interactions or elucidating electron dynamics and structure of complex systems. Here, we uncover a form of simultaneous spin and orbital angular momentum conservation and show, theoretically and experimentally, that this phenomenon allows for unprecedented control over the divergence and polarization of extreme-ultraviolet vortex beams. High harmonics with spin and orbital angular momenta are produced, opening a novel regime of angular momentum conservation that allows for manipulation of the polarization of attosecond pulses-from linear to circular-and for the generation of circularly polarized vortices with tailored orbital angular momentum, including harmonic vortices with the same topological charge as the driving laser beam. Our work paves the way to ultrafast studies of chiral systems using high-harmonic beams with designer spin and orbital angular momentum.

S ince the pioneering studies of Beth $^{1}$ and Allen et al. ${ }^{2}$, it has been well known that propagating light waves, under the paraxial approximation, can possess two distinct forms of angular momenta-spin and orbital angular momentum (SAM and OAM, respectively). Although similar in nature, the distinction between the SAM and OAM of light is straightforward when one considers an interaction picture: the SAM of light is mediated through anisotropic interactions, whereas the OAM of light is associated with inhomogeneity in a physical system ${ }^{3}$. This powerful decoupling allows for the independent manipulation and measurement of either the SAM or OAM of a single light field ${ }^{4-6}$, and enables many applications ${ }^{7}$, including optical sensing and communication ${ }^{8}$, molecular detection ${ }^{9}$, kinematic micromanipulation ${ }^{10}$ and photonic momentum control ${ }^{11}$.

Initially, applications exploiting optical SAM or OAM interactions were largely limited to macroscopic systems using visible light. These limitations stemmed from the challenges in producing and controlling coherent, short-wavelength light beyond the ultraviolet (UV). Fortunately, recent advances in high-harmonic generation (HHG) have bridged this photonic gap, allowing for the straightforward generation of coherent, subfemtosecond radiation in the extreme UV (EUV), with controllable SAM or OAM properties ${ }^{12-27}$. These advanced light sources have opened up the possibility of monitoring and manipulating the SAM and OAM of light-matter interactions on the atomic scale, with the potential of extending quantum optical/logical metrologies, optical manipulation and chiral spectroscopies to the nanometre spatial and subfemtosecond temporal scales.

Fundamentally, these exciting capabilities are enabled by the quantum physics of the high-harmonic upconversion process ${ }^{28,29}$.
In $\mathrm{HHG}$, an electron wavepacket in an atomic, molecular or material system is liberated by an intense laser field, which then accelerates the free electron wavepacket. The oscillatory nature of the laser field can drive the wavepacket back to the parent ion and, on recollision, the acquired kinetic energy is released in the form of high-order harmonics, which can span deep into the EUV and soft X-ray spectral regions ${ }^{30-34}$. It is this field-driven nature of HHG that provides an opportunity for mapping the properties of near-infrared laser light, in particular SAM and OAM, to short-wavelength radiation. Indeed, recent experimental demonstrations of independent control of $\mathrm{SAM}^{13,18,35,36}$ or OAM ${ }^{21,24,37}$ in the EUV via HHG have propelled the topic of optical angular momentum control and measurement to the forefront of attosecond science.

Here, we present a significant advance in producing EUV beams with designer angular momenta (that is, helicity and twist) by generating high-order harmonics-and attosecond pulses-possessing controllable spin and orbital angular momenta. By driving the $\mathrm{HHG}$ process with a bichromatic, counter-rotating vortex beam (that is, a bicircular vortex beam), we uncover and subsequently harness, a novel form of simultaneous SAM-OAM momentum conservation. We exploit this simultaneous conservation to produce spatially isolated vortex beams of opposite SAM through proper selection of the angular momenta of the bicircular vortex driver, which allows us to control the polarization state of attosecond EUV vortex beams in the time domain; from linear to purely circularly polarized. This unique SAM-OAM control also makes it possible to generate highly elliptically polarized high-harmonic OAM beams with controllable topological charge. In particular, apart from generating highly charged vortex beams with designer OAM, we generate harmonic beams of highly circular polarization with the same, low, topological

'JILA - Department of Physics, University of Colorado and NIST, Boulder, CO, USA. ${ }^{2}$ Grupo de Investigación en Aplicaciones del Láser y Fotónica, Departamento de Física Aplicada, University of Salamanca, Salamanca, Spain. ${ }^{3}$ Quantum Electromagnetics Division, National Institute of Standards and Technology, Boulder, CO, USA. 4Present address: Departamento de Química, Universidad Autónoma de Madrid, Madrid, Spain. *e-mail: kevin.dorney@colorado.edu; carloshergar@usal.es 


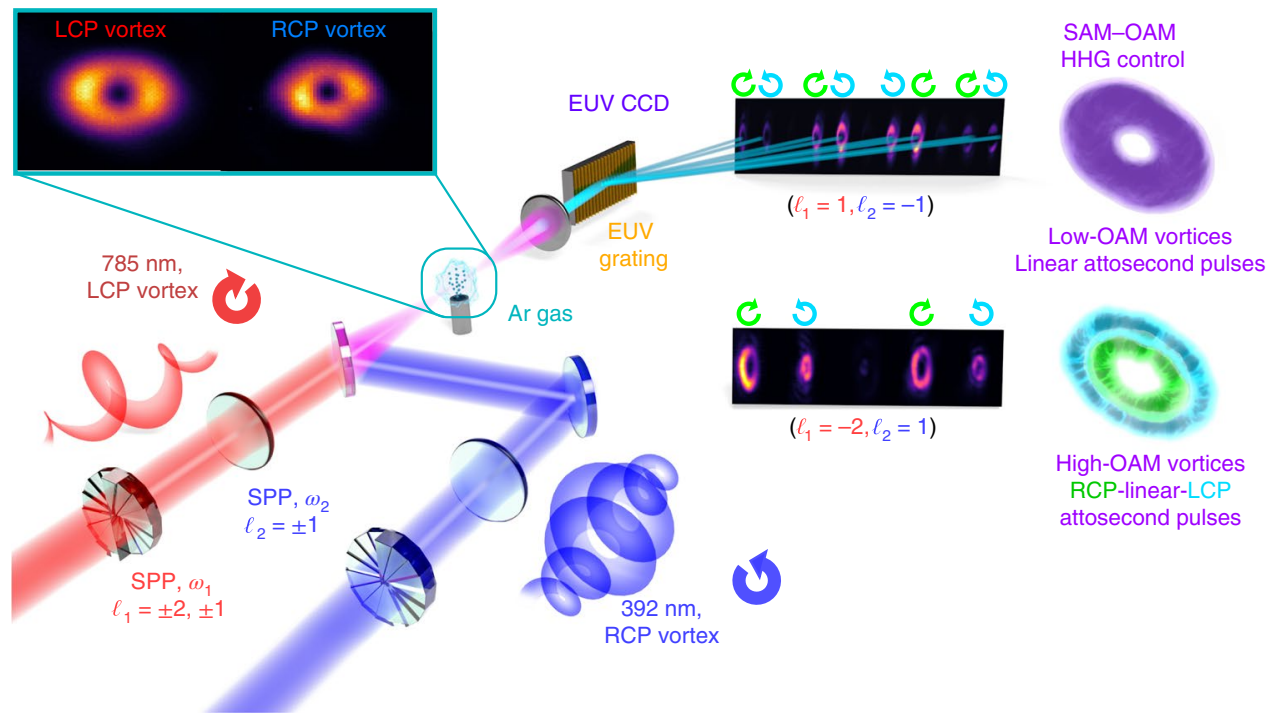

Fig. 1 | Bicircular HHG in the presence of simultaneous SAM-OAM conservation (SAM-OAM HHG). $\mathbf{a}$, In the experiment, circularly polarized beams of opposite helicity are passed through independent spiral phase plates (SPPs) producing high-purity circularly polarized OAM vortex beams (top-left inset). These beams are spatiotemporally overlapped in a supersonic expansion of Ar gas, yielding a bicircular SAM-OAM vortex beam that drives the HHG process. The SAM-OAM EUV beams are collected via a cylindrical mirror-grating pair (cylindrical mirror omitted for clarity) and an EUV camera. The detectors show experimental EUV OAM beam profiles collected in the far field, $8 \mathrm{~cm}$ beyond the flat-field focal plane of the spectrometer. In this scheme, the detectors show experimental SAM-OAM HHG for both complementary $\left(\ell_{1}=1, \ell_{2}=-1\right)$ and non-degenerate $\left(\ell_{1}=-2, \ell_{2}=1\right)$ configurations of the bicircular vortex driver. When driven with complementary OAM beams, OAM harmonics are generated with a low OAM charge, equal to the components of the bicircular vortex (top-right inset). If a non-degenerate vortex driver is employed for the SAM-OAM HHG process, EUV vortices of high-OAM charge and opposite helicity exhibit significantly different OAM charges, which results in RCP and LCP harmonics being spatially separated in the far field (bottom-right inset).

charge-equal to the co-rotating component of the bicircular vortex driving laser field. Our work opens a route to perform ultrafast studies of angular momentum exchange and interactions at EUV/Xray wavelengths, with the potential for nanometre spatial and subfemtosecond temporal resolution.

\section{Results}

Generation of high-harmonic beams with SAM and OAM. The generation of SAM-OAM EUV vortex beams is depicted in Fig. 1. A bichromatic Mach-Zehnder interferometer is used to produce two collinear, vortex laser beams with opposite helicities derived from the fundamental (frequency $\omega_{1}$, spin $\sigma_{1}=-1$, left-circular polarization, LCP, and topological charge $\ell_{1}$ ) and frequency-doubled $\left(\omega_{2}=2 \omega_{1}, \sigma_{1}=+1\right.$, right-circular polarization, RCP, $\left.\ell_{2}\right)$ output of an ultrafast Ti:sapphire amplifier (see Methods and Supplementary Section 1). These beams are then combined, spatially and temporally, to yield a bicircular vortex beam that drives HHG in a supersonic expansion of Ar gas. The emitted harmonics are collected via an EUV spectrometer consisting of a cylindrical mirror-flat grating spectrometer and an EUV charge coupled device (CCD) camera (see Methods). As a reference for these experiments, we have performed full quantum HHG simulations including propagation using the electromagnetic field propagator ${ }^{38}$, a method that was used in several previous calculations of HHG involving either SAM ${ }^{17,18,32,35,39,40}$ or $\mathrm{OAM}^{20,23,26,27,41}$ (see Methods and Supplementary Section 2).

As a first demonstration of this novel, simultaneous SAM-OAM conservation in HHG, we drive the SAM-OAM HHG process with a bicircular vortex laser field with $\ell_{1}=1$ and $\ell_{2}=1$. In this configuration, a high-harmonic spectrum consisting of doublets of EUV vortex beams is generated, where the harmonics in each doublet possess the same topological charge, but opposite helicities (Fig. 2a,b). Most notably, the strong suppression of every third harmonic order confirms that the same SAM conservation rules are upheld in SAM-OAM HHG as in traditional bicircular $\mathrm{HHG}^{42,43}$. SAM conservation, $\sigma_{q}=n_{1} \sigma_{1}+n_{2} \sigma_{2}$, together with the parity constraints, implies that the number of photons absorbed from each of the drivers $\left(n_{1}, n_{2}\right)$ to generate the $q$ th order harmonic must differ by one: $n_{2}=n_{1}-\sigma_{q} \sigma_{1}$. Taking into account photon energy conservation, $q \omega_{1}=n_{1} \omega_{1}+n_{2} \omega_{2}$, the resulting HHG spectrum consists of pairs of adjacent harmonics with opposite circular polarization, and a third, missing harmonic, whose suppression indicates a high circularity of the SAM-OAM EUV beams. This is confirmed by EUV magnetic circular dichroism (EUV MCD) measurements on an $\mathrm{Fe}_{0.75} \mathrm{Co}_{0.25}$ film (Fig. 2d and Methods). Unfortunately, a quantitative value of the harmonic ellipticity cannot be obtained at this time, as MCDderived ellipticities require a comparison with existing synchrotron data and such experimental data does not exist for this energy range. However, a comparison with extrapolated synchrotron data from resonant MCD measurements of Fe films ${ }^{15}$ allows us to confirm that the SAM-OAM HHG harmonics are highly elliptically polarized. In short, the strong non-resonant EUV MCD signal, suppression of spin-forbidden harmonic orders, and the excellent agreement with the theoretical simulations verifies the near-circular polarization of the SAM-OAM EUV vortex beams (see below). We note that depolarization effects are unlikely in this geometry, as the MCD sample is placed far from the generating region, yet a strong MCD signal is still observed.

The simultaneous conservation of SAM and parity in SAMOAM HHG also restricts the allowed OAM values for each harmonic $^{44}$. If both beams are linearly polarized, the topological charge of the $q$ th order harmonic driven by a bichromatic laser field is given by a simple OAM conservation rule $\ell_{q}=n_{1} \ell_{1}+n_{2} \ell_{2}$ (ref. ${ }^{23}$ ). Each harmonic can, therefore, exhibit several OAM contributions depending on the number of photons absorbed from each driver ${ }^{23}$. Note that non-perturbative OAM contributions ${ }^{23}$ do not appear in our SAM-OAM HHG scheme since the intensity distribution at 


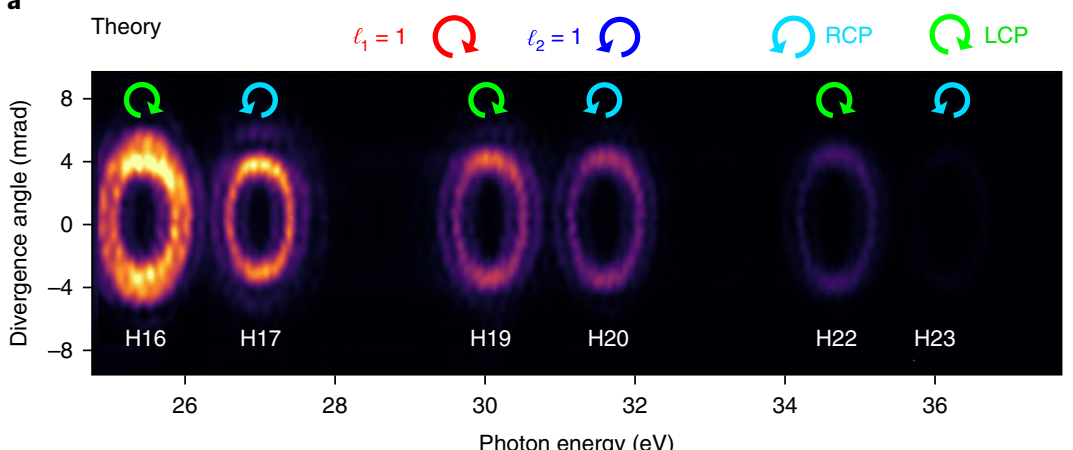

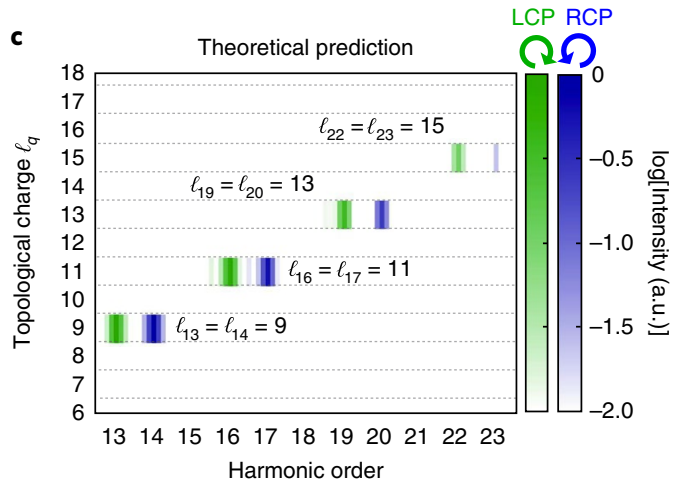

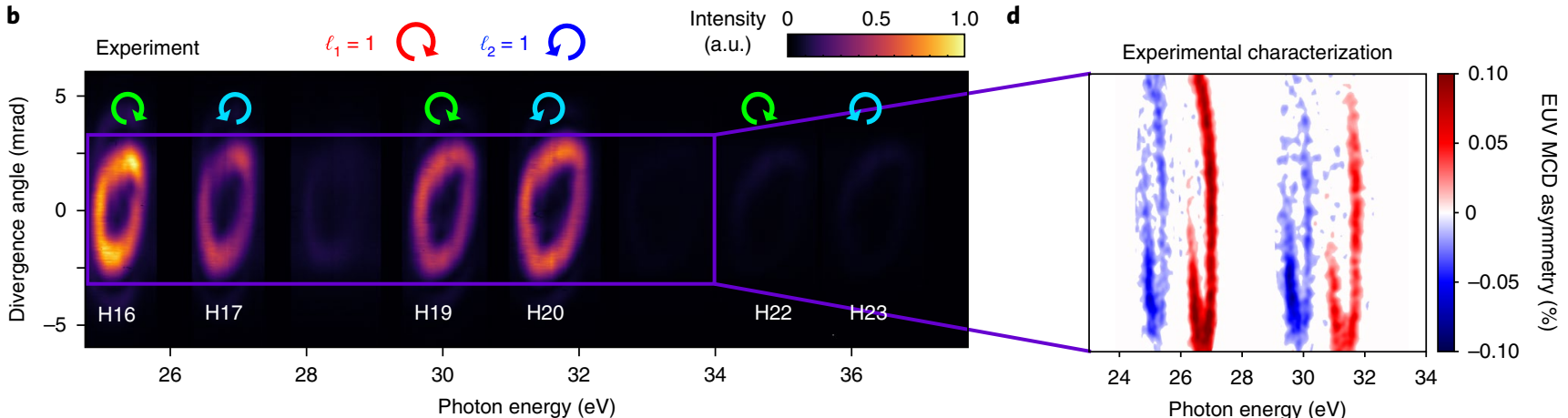

Fig. 2 | Experimental generation and theoretical confirmation of SAM-OAM EUV vortices in the presence of simultaneous SAM-OAM conservation. a, Full quantum simulation results showing spectrally dispersed SAM-OAM harmonics driven by a bicircular vortex driving beam $\left(\sigma_{1}=-1, \sigma_{2}=+1, \ell_{1}=1\right.$, $\left.\ell_{2}=1\right)$. b. Spatio-spectral measurement of SAM-OAM EUV vortex beams produced via HHG from the same configuration of the bicircular driver, exhibiting a clean mode with a clear singularity on axis, a single bright intensity ring and strong suppression of every spin-forbidden (that is, every third) harmonic order. Further calculations (not shown) indicate that the different divergences between the theory (a) and experimental (b) results are due to slightly different driving-beam waists. c, Calculated topological charge of the LCP (green) and RCP (blue) harmonic vortices confirms that the presence of simultaneous SAM-OAM conservation results in neighbouring harmonics possessing a similar OAM spectrum. The OAM is calculated through a Fourier transform along the azimuthal coordinate for each RCP and LCP frequency component. d, EUV MCD measurement of a 20 -nm-thick Fe ${ }_{0.75} \mathrm{Co}_{0.25}$ film confirms the near-circular polarization of the generated SAM-OAM EUV vortices. Note that inhomogeneity in the EUV MCD signal for each harmonic is the result of slight pointing fluctuations and changes in the sample structure for the different magnetizations of the Fe ${ }_{0.75} \mathrm{Co}_{0.25}$ film.

focus does not vary azimuthally, as also observed in non-collinear bichromatic OAM HHG ${ }^{24,25}$. However, combining the above-mentioned SAM and OAM conservation rules in bicircular SAM-OAM HHG, the topological charge of the $q$ th order harmonic satisfies

$$
\ell_{q}=\frac{q+2 \sigma_{q} \sigma_{1}}{3}\left(\ell_{1}+\ell_{2}\right)-\sigma_{q} \sigma_{1} \ell_{2}
$$

where SAM conservation restricts $\ell_{q}$ to a single value. The products $\sigma_{2,1} \ell_{1,2}$ show that the SAM and OAM of the bicircular vortex driver are inherently entwined via the HHG process, which connects the SAM and OAM of the harmonics to those of the driving beams. This manifests as an entirely new form of simultaneous conservation of SAM and OAM, where the OAM $\left(\ell_{q}\right)$ and SAM $\left(\sigma_{q}\right)$ of each harmonic vortex can be controlled via the interplay of the $\operatorname{SAM}\left(\sigma_{1}, \sigma_{2}\right)$ and the OAM $\left(\ell_{1}, \ell_{2}\right)$ of the drivers. At this point we would like to remark that the SAM and OAM of the harmonics are controlled via simultaneous conservation of these quantities during the HHG process, and that SAM and OAM are not converted from one another as in more traditional SAM-OAM coupling observed in subwavelength and non-paraxial optical regimes ${ }^{45}$.

The effects of simultaneous SAM-OAM conservation are readily evident by comparing the theoretical (Fig. 2a) and experimental (Fig. 2b) SAM-OAM EUV spectra. In such a configuration, $\left(\ell_{1}=\ell_{2}=1\right)$, the OAM conservation rule, equation (1), reads as $\ell_{q}=\left(2 q+\sigma_{q} \sigma_{1}\right) / 3$. This implies that each pair of adjacent harmonics exhibits the same OAM, as can be seen in Fig. 2c, where the OAM is calculated by performing a Fourier transform along the azimuthal coordinate for each frequency component ${ }^{23}$. This method of determining the OAM content is ideally suited for arbitrary, structured beams possessing OAM as it does not rely on decomposition into a particular basis set and is thus more general than methods employing modal decomposition. We note that each harmonic in the SAM-OAM EUV spectrum has a uniform azimuthal intensity profile and little radial mode character, indicating similar modal content between the experimental and theoretical SAM-OAM HHG. Moreover, the excellent agreement of the beam profiles between the experiment and theory also suggests a highly elliptical polarization of the harmonics; if depolarization effects were present, the SAM-dependent OAM selection rules would be relaxed, leading to a superposition of topological charges in each harmonic and consequently nonuniform SAM-OAM HHG beam profiles ${ }^{23}$. Although the OAM of the experimental harmonics was not measured in this work, the points mentioned above strongly suggest a similar modal content as that obtained in the theoretical spectra. Taken together, the generated SAM-OAM EUV vortex beams possess both a high modal purity as well as near-circular polarization.

Polarization control of attosecond EUV pulses through simultaneous SAM-OAM conservation. In the past decade, there has been an explosion of interest in controlling the spin polarization of attosecond EUV waveforms, as full polarization control would allow for 

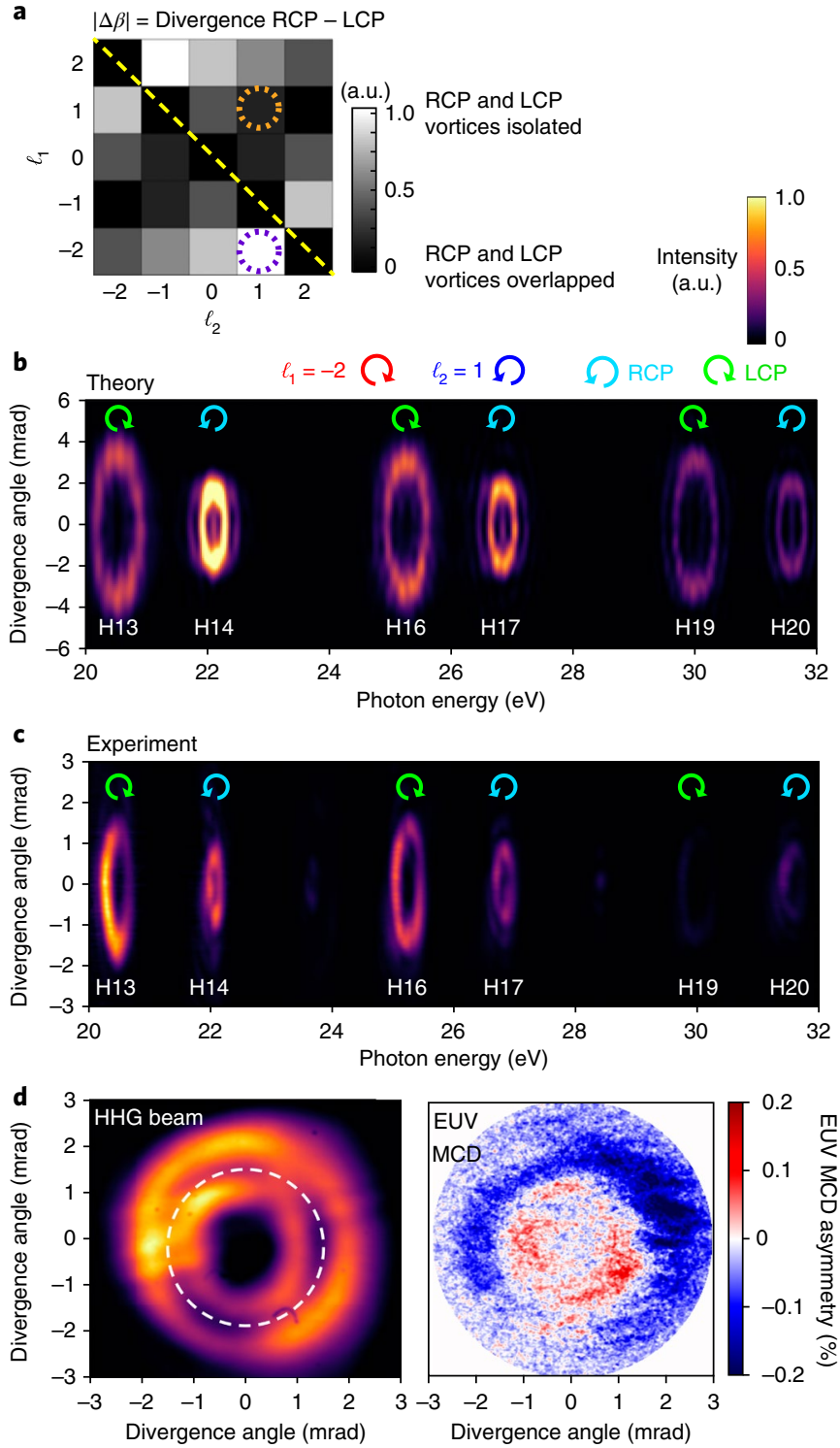

Fig. 3 | Separation of EUV high-harmonic vortex beams with opposite circularities through the OAM of the bicircular vortex driver. a, Theoretical estimation for the divergence difference between the RCP and LCP harmonics $(|\Delta \beta|)$ as a function of the OAM of the constituents of the bicircular vortex field. The dashed circles correspond to configurations of the bicircular vortex drive used to generate the experimental SAM-OAM EUV harmonics (orange dashed circle shows OAM case with $\ell_{1}=\ell_{2}=1$ in Fig. 2 , purple dashed circle shows OAM case with $\ell_{1}=-2, \ell_{2}=1$, and yellow dashed line shows the case for opposite OAMs of the driving fields, see main text for details). b, A full quantum simulation, including propagation, shows that when SAM-OAM $\mathrm{HHG}$ is driven with a bicircular OAM driver of $\ell_{1}=-2, \ell_{2}=1$, the RCP and LCP vortices exhibit significantly different divergence, resulting in spatially separated RCP and LCP beams in the far field. Further calculations (not shown) indicate that differences in the theoretical and experimental spectra are due to slightly different driving beam parameters. c, A spatio-spectral measurement of SAM-OAM EUV harmonics under the same configuration of the bicircular vortex driver as in $\mathbf{b}$, which confirms the OAM-dependent divergence control afforded by SAM-OAM HHG. d, This control results in a dual intensity ring structure in the far field of the HHG beams (left, dashed circle highlights the intensity null between the vortices), where the inner, RCP beam is entirely contained by the LCP vortex beam, as confirmed by a spatially resolved EUV MCD measurement (right). The inhomogeneity observed in the EUV MCD-retrieved beam profiles is the result of slight fluctuations in beam pointing, microstructuring of the MCD sample, and attenuation of low-intensity regions of the beam. the possibility of custom-tailored harmonic beams for attosecond, nanometric chiral spectroscopies and metrologies. As such, a wide variety of experimental ${ }^{12-18,32,35,36,40}$ and theoretical ${ }^{41,46-48}$ schemes have been investigated to control the SAM of EUV high harmonics. Here we show that the interplay of SAM-OAM conservation inherent to SAM-OAM HHG opens a route to control the polarization of attosecond pulses.

In traditional bicircular HHG driven by collinear pulses with a Gaussian spatial profile, RCP and LCP harmonics spatially overlap in the far field, yielding attosecond bursts with a rotating, linear polarization in the time domain ${ }^{39,43,46,48,49}$. This is largely the result of the similar propagation properties between RCP and LCP harmonics, as the divergence of a light field does not typically depend on its SAM. Fortunately, recent advances in bicircular HHG have shown that elliptically polarized attosecond pulse trains (APTs) ${ }^{35,36}$, spatially isolated APTs ${ }^{15}$ and isolated attosecond pulses ${ }^{18}$ of opposite helicity can be generated and the ellipticity can be fully tuned by the parameters of the visible driving lasers. Although elegant, these methodologies are only capable of controlling either the SAM or the divergence of the EUV harmonics, instead of both simultaneously. However, the simultaneous conservation of SAM and OAM in bicircular SAM-OAM HHG, together with the OAM-dependent divergence inherent to vortex beams, allow us to circumvent these limitations and simultaneously control the divergence of EUV light and the polarization of the underlying APTs.

To show the power of this concept, we consider HHG driven by a single-mode OAM driver. It is known that the divergence of a harmonic beam decreases with the harmonic order, while the divergence of a vortex beam increases with its topological charge. Therefore, for a single-mode OAM driver, the simple OAM conservation rule, $\ell_{q}=q \ell_{1}$, results in a HHG spectrum where all harmonics are emitted with a similar divergence ${ }^{20-22,41}$. In the case of bicircular SAM-OAM HHG, the restricted selection rules resulting from the mixing of SAM and OAM can be exploited to control the divergence of the harmonics and, for example, to yield spatially isolated vortex beams of pure RCP and LCP polarization, something that is not possible with either linear OAM HHG or collinear, bicircular HHG with Gaussian drivers. This point is illustrated below, where we employ a simple theoretical analysis based on Fraunhofer diffraction that predicts the divergence difference between the RCP and LCP harmonics $(\Delta \beta)$ as a function of the OAMs of the bicircular vortex driver (see Supplementary Section 4 ) as

$$
\Delta \beta \propto\left(\ell_{1}-2 \ell_{2}\right) \frac{\left|\ell_{1}+\ell_{2}\right|}{\ell_{1}+\ell_{2}}
$$

where $\Delta \beta=0$ if $\ell_{1}+\ell_{2}=0$, and we are assuming $\omega_{2} / \omega_{1}=2$. In Fig. 3a, we plot the relative values of $|\Delta \beta|$ as a function of $\ell_{1}$ and $\ell_{2}$. The divergence difference between RCP and LCP harmonics depends strongly on the choice of $\ell_{1}$ and $\ell_{2}$, and as a general trend, the greatest differences in divergence correspond to large differences in the OAMs of the drivers. Thus, it is the OAM-dependent divergence, combined with the simultaneous angular momentum selection rules induced by the bicircular OAM driver, that gives control over the spatial distribution of RCP and LCP harmonics in the EUV vortex beam, and as a consequence, over the polarization of the attosecond pulses.

To further illustrate this point, we return to the case of $\ell_{1}=\ell_{2}=1$, presented in Fig. 2 (orange dashed circle in Fig. 3a). The RCP and LCP vortices exhibit similar divergences and thus spatially overlap, yielding linearly polarized APTs in the time domain. However, closer inspection of the divergence analysis shows that spatial separation of RCP and LCP components of the EUV beams is achieved when the OAMs of the drivers are complementary, but different in magnitude. To demonstrate this unique capability, we drive the 

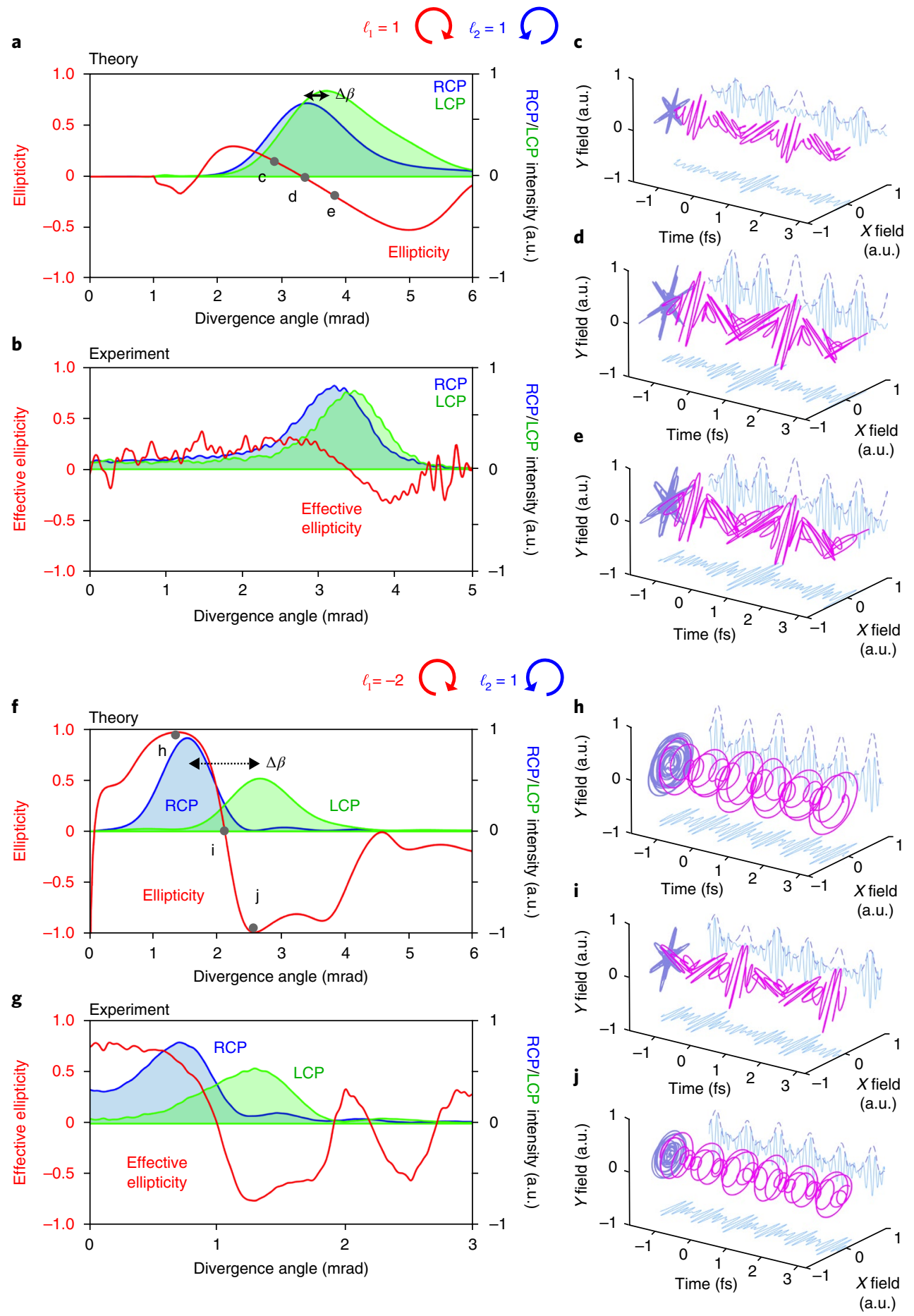

Fig. 4 | Control over the OAMs of the bicircular driver yields full control of the polarization state of APTs in SAM-OAM HHG. a-e, Spectrally integrated intensities of RCP (blue) and LCP (green) harmonics (from the 13th to the 23rd) for a degenerate bicircular vortex $\left(\ell_{1}=\ell_{2}=1\right.$ ) confirm that SAM-OAM high harmonics possess similar divergences and thus overlap spatially in the far field (theory (a) and experiment (b)), resulting in predominately linearly polarized APTs across the EUV vortex beam profile, as shown in the simulated temporal evolution detected at 2.7 (c), 3.3 (d) and 3.7 (e) mrad (magenta lines show full APT, while the blue and purple lines show component projections in the $X T, Y T$ and $X Y$ planes, respectively). $\mathbf{f}-\mathbf{j}$, When SAM-OAM HHG is driven with a non-degenerate bicircular vortex beam $\left(\ell_{1}=-2, \ell_{2}=1\right)$, the RCP and LCP harmonics exhibit significantly different divergences, which results in the spatial separation of RCP and LCP vortices in the far field (theory $(\mathbf{f})$ and experiment $(\mathbf{g})$ ). The combined theoretical and experimental results confirm the degree of divergence control via simultaneous spin-orbit conservation in the HHG process, which results in a spatial evolution of the polarization of the APTs from RCP (h, 1.4 mrad), to linear (i, $2.1 \mathrm{mrad})$, to LCP (j, $2.6 \mathrm{mrad})$.

HHG process with a non-degenerate, complementary, bicircular OAM driver $\left(\ell_{1}=-2, \ell_{2}=1\right.$, purple dashed circle in Fig. $\left.3 a\right)$. The theoretical and experimental SAM-OAM HHG spectra (Fig. 3b,c) clearly confirm the ramifications of SAM-OAM conservation on controlling the divergence of the EUV vortices; RCP vortex harmonics are entirely contained by their LCP counterparts, resulting 
a

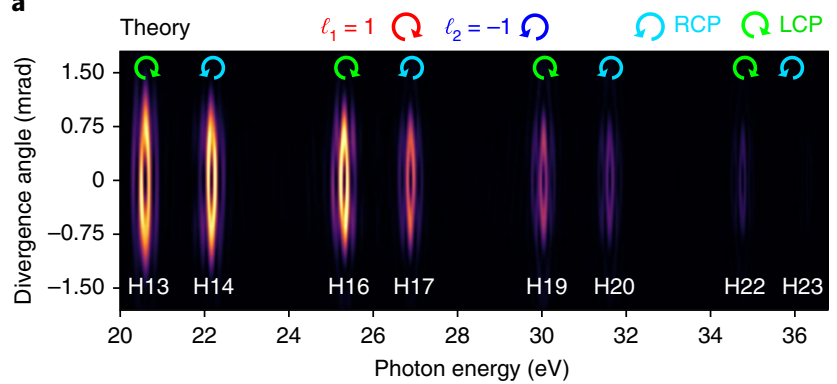

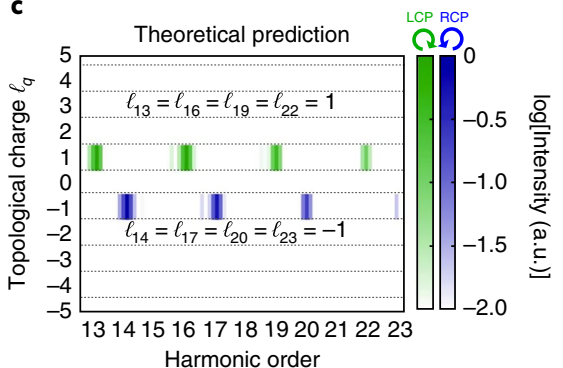

d b

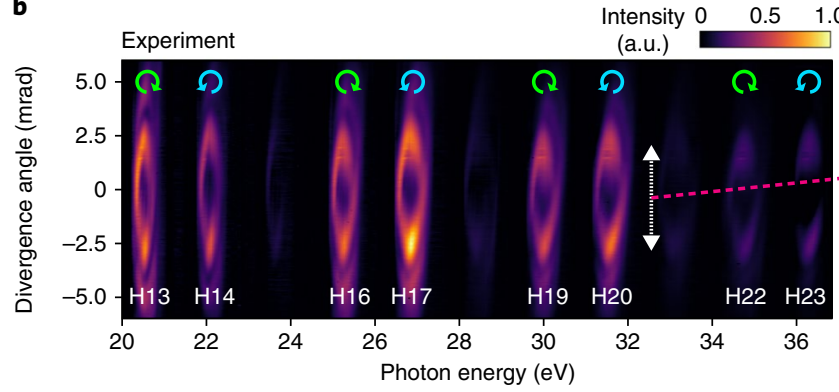

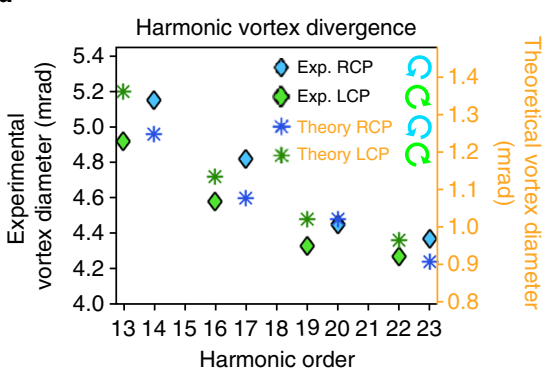

Fig. 5 | Circularly polarized high-harmonic vortex beams with equal, low-charge OAM. a,b, Simulated (a) and experimental (b) high-harmonic spectra of the EUV OAM beams driven by a degenerate, complementary bicircular field $\left(\ell_{1}=1, \ell_{2}=-1\right)$, exhibiting pairs of high harmonics of alternating helicity. The decrease in divergence of the harmonics as a function of energy, combined with the localized singularity indicate that each harmonic has a similar, low topological charge. c,d, Theoretical OAM content of the LCP (green) and RCP (blue) HHG projections, which shows that all harmonics with the same SAM present the same OAM as the driver with that SAM. The manifestation of the low topological charge of the EUV harmonics results in a decrease of the angular diameter of the beams as a function of energy, as clearly demonstrated in d, where experimental (theoretical) results are shown by diamonds (asterisks). Further calculations (not shown) indicate that the different divergences between the theory and experimental results are due to slightly different driving beam parameters (that is, size of beam waist and the exact location of beam waists), which also results in a low-intensity secondary intensity ring in the experimental spectra.

in a double-ring structure in the far field (Fig. 3d, left). The nearcircular polarization of the double-ring vortex beam is confirmed by EUV MCD measurements, which reveal two distinct intensity rings of opposite helicity (Fig. 3d, right).

The effects of the OAM-dependent divergence control in SAMOAM HHG-and thus the OAM-based control over the polarization of the attosecond pulses-can be more clearly demonstrated by performing a spectral integration across the SAM-OAM HHG spectra. Returning to a degenerate configuration of the driving field $\left(\ell_{1}=1, \ell_{2}=1\right)$, the RCP and LCP harmonics both exhibit similar divergences, as well as comparable spectral intensities (Fig. 4a,b). If we define the ellipticity of the attosecond pulses as $\varepsilon=\left(I_{\mathrm{RCP}}-I_{\mathrm{LCP}}\right) /\left(I_{\mathrm{RCP}}+I_{\mathrm{LCP}}\right)$, where $I_{\mathrm{RCP}}$ and $I_{\mathrm{LCP}}$ are the intensities of the RCP and LCP harmonics, the spatial overlap of the RCP and LCP components of the theoretical SAM-OAM HHG spectrum results in attosecond pulses with predominate linear polarization in the time domain (Fig. 4c-e). Given the excellent agreement between the theoretical and experimental spectra and spectrally integrated signals, it is highly likely that the experimental harmonics possess similar ellipticities, and thus we expect a similar polarization of the experimental APTs. However, our experimental and theoretical SAM-OAM HHG spectra show that this symmetry can be broken by employing a complementary, non-degenerate vortex driver (for example, $\ell_{1}=-2, \ell_{2}=1$ ). In this configuration, the oppositely polarized vortex beams experience large enough divergences to be spatially isolated (Fig. 4f,g). As such, the ellipticity of the attosecond pulses evolves all the way from near right circular $(\varepsilon \approx 1)$, to linear $(\varepsilon=0)$, to near left circular $(\varepsilon \approx-1)$ across the EUV beam profile (Fig. $4 \mathrm{~h}-\mathrm{j}$ ). Therefore, by properly modifying the OAM of the driving fields, we can spatially control the ellipticity of the attosecond pulses and this degree of control becomes even greater as the difference in OAM of the bicircular driver increases, where pure circular attosecond pulses $(\varepsilon= \pm 1)$ can be obtained (see Supplementary Section 4).

High-harmonic vortices with circular polarization and identical low-charge OAM. Since the first experiments of HHG driven by OAM beams ${ }^{19}$, there has been significant interest in controlling the OAM content of the high harmonics, as well as generating harmonic vortices with a low topological charge ${ }^{24,25}$. Such a desire is rather pragmatic: many OAM light-matter interactions depend on the topological charge of the optical vortex beam, and lower topological charges inherently lead to less complex interactions and detection geometries. Unfortunately, the simple OAM conservation law governing HHG driven by single OAM beams, $\ell_{q}=q \ell_{1}$, implies that high-order harmonic OAM beams emerge with highly charged $\mathrm{OAM}^{20-23,39,50}$. Recently, this limitation was overcome by employing a non-collinear scheme ${ }^{24,25}$, resulting in linearly polarized EUV vortex beams with low OAM charge. Here we demonstrate that the simultaneous conservation of SAM and OAM in bicircular SAMOAM HHG can yield high harmonics not only with high topological charge (as shown in the previous sections), but also with the same OAM as the driving field, without the complications induced by a non-collinear geometry.

When the OAM of the drivers fulfil $\ell_{1}=-\ell_{2}$, equation (1) transforms into $\ell_{q}=-\sigma_{q} \sigma_{1} \ell_{2}$, which indicates that all RCP (LCP) harmonics exhibit the same topological charge as the RCP (LCP) driving field (see Supplementary Section 4). This implies that the divergence decreases linearly with the harmonic order and that RCP and LCP harmonics exhibit similar divergence $(\Delta \beta \approx 0$, yellow dashed line in Fig. 3a). As a final demonstration of the control afforded by the conservation rules in SAM-OAM HHG, in Fig. 5a,b we present the 
theoretical and experimental HHG spectra generated by a bicircular driver of complementary topological charge $\left(\ell_{1}=1, \ell_{2}=-1\right)$. Most notably, the divergence decreases with the harmonic order, which is a clear indication of the equal OAM of the harmonics, as shorter-wavelength harmonics have a lower divergence given the same topological charge. This fact is further corroborated by considering the highly circular polarization of the EUV beams. If the harmonics were not circularly polarized, the lack of SAM conservation would result in the generation of highly divergent, highly charged OAM HHG beams, as implied by the simple OAM conservation rule $\ell_{q}=n_{1} \ell_{1}+n_{2} \ell_{2}\left(\right.$ ref. $\left.{ }^{23}\right)$. However, our SAM-OAM HHG scheme demands SAM conservation, which results in LCP harmonics emitted with $\ell_{\mathrm{LCP}}=\ell_{1}=1$, and RCP harmonics with $\ell_{\mathrm{RCP}}=\ell_{2}=-1$ (Fig. $5 \mathrm{c}$ ). The physical manifestation of this effect is a decrease in the angular diameter of the SAMOAM harmonics as a function of harmonic energy, which is captured in both experiment and theory (Fig. 5d). Although the angular divergence of the experimental harmonics within each doublet show an opposite behaviour with respect to the theoretical spectra (a result of slight differences in beam parameters between the experiment and simulations), the general trend is clearly observed. Taken together, our observations demonstrate the power of SAM-OAM HHG for complete control of the angular momentum of attosecond, structured EUV beams carrying OAM.

\section{Discussion}

Despite the fact that many light-matter interactions can possess independent SAM and OAM conservation laws, the simultaneous conservation of both quantities has rarely been utilized to control the light-matter interaction, particularly in HHG. Here, we have shown that the simultaneous SAM-OAM conservation in highly nonlinear and intense field interactions can be used as a powerful tool for controlling the upconversion process, and producing HHG beams with designer SAM and OAM. By constructing a bicircular vortex driving beam with varying topological charges of the constituent waveforms, we showed that SAM-OAM HHG entwines the angular momentum conservation rules, yielding unprecedented and exquisite control of the SAM, OAM and the divergence of EUV light fields.

To demonstrate the potential of the simultaneous SAM-OAM conservation in this regime, we experimentally demonstrated three distinct mixing cases where (1) circularly polarized high-harmonic vortex beams are generated with a similar divergence, and thus similar propagation properties regardless of spin polarization or photon energy; (2) spatially isolated harmonic vortex beams are produced, allowing spatial tuning of the polarization of attosecond pulseslinear, elliptical or pure RCP/LCP-which enables spatially resolved dichroism measurements or isolation of an EUV vortex beam of pure RCP or LCP polarization; and (3) EUV harmonic vortex beams with topological charge equal to, and thus controlled by, the OAM of the driving lasers. These results significantly extend the degree of the control over short-wavelength radiation via manipulation of visible driving lasers. Perhaps most importantly, our results demonstrate the simultaneous optical spin-orbit angular momentum conservation as a gateway to an entirely new set of applications and fundamental investigations. These exciting applications are enabled by the short wavelength of high harmonics, which allows these designer SAM-OAM beams to be focused to scale lengths comparable to nanostructured and molecular systems, facilitating new nanometric spectroscopies and opportunities for nanoscale optical manipulation. Aside from these capabilities, we also add a degree of freedom, the OAM, to the recent experiments that uncovered ultrafast spin/charge dynamics in magnetic materials ${ }^{51-54}$ and the ultrafast study of chiral molecular systems ${ }^{9,55}$. One could also envision the possibility for skyrmionic spectroscopies ${ }^{56,57}$, or for taking advantage of SAM-OAM selection rules in photoionization/photoemission experiments ${ }^{58,59}$, all of which do not necessarily demand nanometre-scale focusing conditions. Finally, circularly polarized short-wavelength OAM beams also open up new scenarios in spatially resolved circular dichroism spectroscopies (as demonstrated in this work), and even the possibility of performing SAM and/or OAM dichroism X-ray absorption measurements, which allows for decoupling the effects of orbital and spin contributions to energy transport ${ }^{60}$.

Note added in proof: We note that while this work was under review, a theoretical work ${ }^{44}$ was published describing the selection rules in SAM-OAM HHG.

\section{Online content}

Any methods, additional references, Nature Research reporting summaries, source data, statements of data availability and associated accession codes are available at https://doi.org/10.1038/ s41566-018-0304-3.

Received: 29 May 2018; Accepted: 25 October 2018; Published online: 10 December 2018

\section{References}

1. Beth, R. A. Mechanical detection and measurement of the angular momentum of light. Phys. Rev. 50, 115-125 (1936).

2. Allen, L., Beijersbergen, M. W., Spreeuw, R. J. C. \& Woerdman, J. P. Orbital angular momentum of light the transformation of Laguerre-Gaussian laser modes. Phys. Rev. A 45, 8185-8189 (1992).

3. Yao, A. M. \& Padgett, M. J. Orbital angular momentum: origins, behavior and applications. Adv. Opt. Photon. 3, 161-204 (2011).

4. Calvo, G. et al. Measuring the complete transverse spatial mode spectrum of a wave field. Phys. Rev. Lett. 100, 173902 (2008).

5. Ballantine, K. E., Donegan, J. F. \& Eastham, P. R. There are many ways to spin a photon: half-quantization of a total optical angular momentum. Sci. Adv. 2, e1501748 (2016).

6. Cardano, F. \& Marrucci, L. Spin-orbit photonics. Nat. Photon. 9, 776-778 (2015)

7. Willner, A. E. et al. Optical communication using orbital angular momentum beams. Adv. Opt. Photon. 7, 66-106 (2015).

8. Torres, J. P. \& Torner, L. Twisted Photons: Applications of Light with Orbital Angular Momentum (Wiley-VCH, Bristol, 2011)

9. Beaulieu, S. et al. Photoexcitation circular dichroism in chiral molecules. Nat. Phys. 14, 484-489 (2018).

10. Padgett, M. \& Bowman, R. Tweezers with a twist. Nat. Photon. 5, 343-348 (2011)

11. Marrucci, L., Manzo, C. \& Paparo, D. Optical spin-to-orbital angular momentum conversion in inhomogeneous anisotropic media. Phys. Rev. Lett. 96, 163905 (2006).

12. Eichmann, H. et al. Polarization-dependent high-order two-color mixing. Phys. Rev. A 51, R3414 (1995).

13. Fleischer, A., Kfir, O., Diskin, T., Sidorenko, P. \& Cohen, O. Spin angular momentum and tunable polarization in high-harmonic generation. Nat. Photon. 8, 543-549 (2014).

14. Ferré, A. et al. A table-top ultraviolet light source in the extreme ultraviolet for circular dichroism experiments. Nat. Photon. 9, 93-98 (2015).

15. Hickstein, D. D. et al. Non-collinear generation of angularly isolated circularly polarized high harmonics. Nat. Photon. 9, 743-750 (2015).

16. Lambert, G. et al. Towards enabling femtosecond helicity-dependent spectroscopy with high-harmonic sources. Nat. Commun. 6, 6167 (2015).

17. Ellis, J. L. et al. High harmonics with spatially varying ellipticity. Optica $\mathbf{5}$, 479-485 (2018)

18. Huang, P.-C. et al. Polarization control of isolated high-harmonic pulses. Nat. Photon. 12, 349-354 (2018).

19. Zürch, M. et al. Strong-field physics with singular light beams. Nat. Phys. 8, 743-746 (2012)

20. Hernández-García, C. et al. Attosecond extreme ultraviolet vortices from high-order harmonic generation. Phys. Rev. Lett. 111, 083602 (2013).

21. Gariepy, G. et al. Creating high-harmonic beams with controlled orbital angular momentum. Phys. Rev. Lett. 113, 153901 (2014).

22. Géneaux, R. et al. Synthesis and characterization of attosecond light vortices in the extreme ultraviolet. Nat. Commun. 7, 12583 (2016).

23. Rego, L., San Román, J., Picón, A., Plaja, L. \& Hernández-García, C. Nonperturbative twist in the generation of extreme-ultraviolet vortex beams. Phys. Rev. Lett. 117, 163202 (2016).

24. Kong, F. et al. Controlling the orbital angular momentum of high harmonic vortices. Nat. Commun. 8, 14970 (2017).

25. Gauthier, D. et al. Tunable orbital angular momentum in high-harmonic generation. Nat. Commun. 8, 14971 (2017). 
26. Turpin, A., Rego, L., Picon, L., Roman, J. S. \& Hernandez-Garcia, C. Extreme ultraviolet fractional orbital angular momentum beams from high harmonic generation. Sci. Rep. 7, 43888 (2017).

27. Hernández-García, C. et al. Extreme ultraviolet vector beams driven by infrared lasers. Optica 4, 520-526 (2017).

28. Schafer, K. J., Yang, B., DiMauro, L. F. \& Kulander, K. C. Above threshold ionization beyond the high harmonic cutoff. Phys. Rev. Lett. 70, 1599-1602 (1993).

29. Corkum, P. B. Plasma perspective on strong-field multiphoton ionization. Phys. Rev. Lett. 71, 1994-1997 (1993).

30. McPherson, A. et al. Studies of multiphoton production of vacuum-ultraviolet radiation in the rare gases. J. Opt. Soc. Am. B 4, 595 (1997).

31. Ferray, M. et al. Multiple-harmonic conversion of 1064-nm radiation in the rare gases. J. Phys. B 21, L31-L35 (1998)

32. Fan, T. et al. Bright circularly polarized soft X-ray high harmonics for X-ray magnetic circular dichroism. Proc. Natl Acad. Sci. USA 111, 14206-14211 (2015).

33. Rundquist, A. et al. Phase matching of soft X-ray harmonic emission in hollow-core fibers. Science 280, 1412-1415 (1998).

34. Popmintchev, T. et al. Bright coherent ultrahigh harmonics in the keV X-ray regime from mid-infrared femtosecond lasers. Science 336, 1287-1291 (2012).

35. Dorney, K. M. et al. Helicity-selective enhancement and polarization control of attosecond high-harmonic waveforms driven by bichromatic circularly polarized laser fields. Phys. Rev. Lett. 119, 063201 (2017).

36. Jiménez-Galán, Á. et al. Control of attosecond light polarization in two-color bicircular fields. Phys. Rev. A 97, 023409 (2018).

37. Hernández-García, C. A twist in coherent X-rays. Nat. Phys. 13, 327-329 (2017)

38. Hernández-García, C. et al. High-order harmonic propagation in gases within the discrete dipole approximation. Phys. Rev. A 82, 033432 (2010).

39. Chen, C. et al. Tomographic reconstruction of circularly polarized highharmonic fields: 3D attosecond metrology. Sci. Adv. 2, e1501333 (2016)

40. Hernández-García, C. et al. Schemes for generation of isolated attosecond pulses of pure circular polarization. Phys. Rev. A 93, 043855 (2016).

41. Hernández-García, C., San Román, J., Plaja, L. \& Picón, A. Quantum-path signatures in attosecond helical beams driven by optical vortices. New J. Phys. 17, 093029 (2015).

42. Alon, O. E., Averbukh, V. \& Moisevev, N. Selection rules for the high harmonic generation spectra. Phys. Rev. Lett. 80, 3743-3746 (1998).

43. Pisanty, E., Sukiasyan, S. \& Ivanov, M. Spin conservation in high-order harmonic generation using bicircular fields. Phys. Rev. A 90, 043829 (2014).

44. Paufler, W., Böning, B. \& Fritzsche, S. Tailored orbital angular momentum in high-order harmonic generation with bicircular Laguerre-Gaussian beams. Phys. Rev. A 98, 011401(R) (2018).

45. Bliokh, K. Y., Rodríguez-Fortuño, F. J., Nori, F. \& Zayats, A. V. Spin-orbit interactions of light. Nat. Photon. 9, 796-808 (2015).

46. Milošević, D. B., Becker, W. \& Kopold, R. Generation of circularly polarized high-order harmonics by two-color coplanar field mixing. Phys. Rev. A 61, 063403 (2000)

47. Medišauskas, L., Wragg, J., van der Hart, H. \& Ivanov, M. Y. Generating elliptically polarized attosecond pulses using bichromatic counterrotating circularly polarized laser fields. Phys. Rev. Lett. 115, 153001 (2015).

48. Milošević, D. B. Generation of elliptically polarized attosecond pulse trains. Opt. Lett. 40, 2381-2384 (2015).

49. Milošević, D. B. \& Becker, W. Attosecond pulse trains with unusual nonlinear polarization. Phys. Rev. A 62, 011403(R) (2000).

50. Sanson, F. et al. Hartmann wavefront sensor characterization of a high charge vortex beam in the XUV spectral range. Opt. Lett. 43, 2780-2783 (2018).

51. Beaurepaire, E., Merle, J.-C., Daunois, A. \& Bigot, J.-Y. Ultrafast spin dynamics in ferromagnetic nickel. Phys. Rev. Lett. 76, 4250-4253 (1996).

52. Boeglin, C. et al. Distinguishing the ultrafast dynamics of spin and orbital moments in solids. Nature 465, 458-461 (2010).

53. Chen, C. et al. Distinguishing attosecond electron-electron scattering and screening in transition metals. Proc. Natl Acad. Sci. USA 114 E5300-E5307 (2017)
54. Tengdin, P. et al. Critical behavior within 20 fs drives the out-of-equilibrium laser-induced magnetic phase transition in nickel. Sci. Adv. 4, 9744 (2018).

55. Cireasa, R. et al. Probing molecular chirality on a sub-femtosecond timescale Nat. Phys. 11, 654-658 (2015).

56. Fujita, H. \& Sato, M. Ultrafast generation of skyrmionics defects with vortex beams: printing laser profiles on magnets. Phys. Rev. B 95, 054421 (2017).

57. van Veenendaal, $M$. Interaction between $X$-ray and magnetic vortices. Phys. Rev. B 92, 245116 (2015).

58. Picón, A. et al. Transferring orbital and spin angular momenta of light to atoms. New J. Phys. 12, 083053 (2010).

59. Eckart, S. et al. Ultrafast preparation and detection of ring currents in single atoms. Nat. Phys. 14, 701-704 (2018).

60. van Veenendaal, M. \& McNulty, I. Prediction of strong dichroism induced by $\mathrm{X}$ rays carrying orbital angular momentum. Phys. Rev. Lett. 98, 157401 (2007).

\section{Acknowledgements}

The authors are thankful for useful and productive conversations with E. Pisanty, C. Durfee, D. Hickstein, S. Alperin and M. Siemens. H.C.K. and M.M.M. graciously acknowledge support from the Department of Energy BES Award No. DE-FG0299ER14982 for the experimental implementation, as well as a MURI grant from the Air Force Office of Scientific Research under Award No. FA9550-16-1-0121 for the theory. J.L.E., N.J.B. and Q.L.N. acknowledge support from National Science Foundation Graduate Research Fellowships (Grant No. DGE-1144083). C.H.-G., J.S.R. and L.P. acknowledge support from Junta de Castilla y León (SA046U16) and Ministerio de Economía y Competitividad (FIS2013-44174-P, FIS2016-75652-P). C.H.-G. acknowledges support from a 2017 Leonardo Grant for Researchers and Cultural Creators, BBVA Foundation. L.R. acknowledges support from Ministerio de Educación, Cultura y Deporte (FPU16/02591). A.P. acknowledges support from the Marie Sklodowska-Curie Grant, Agreement No. 702565. We thankfully acknowledge the computer resources at MareNostrum and the technical support provided by Barcelona Supercomputing Center (RES-AECT-2014-2-0085). This research made use of the high-performance computingresources of the Castilla y León Supercomputing Center (SCAYLE, www.scayle.es),financed by the European Regional Development Fund (ERDF). Certain commercial instruments are identified to specify the experimental study adequately. This does not imply endorsement by the National Institute of Standards and Technology (NIST) or that the instruments are the best available for the purpose.

\section{Author contributions}

C.H.-G., K.M.D., M.M.M., H.C.K., L.R. and L.P. conceived and designed the SAM-OAM HHG experiment. K.M.D., N.J.B., C.-T.L., J.L.E. and Q.L.N. conducted the experiment. K.M.D. analysed the experimental data. C.H.-G., L.R., J.S.R., A.P. and L.P. performed the theoretical simulations and analysed the resulting data. J.M.S. prepared the EUV MCD sample. C.H.-G., L.P., M.M.M. and H.C.K. supervised the theoretical simulations and experimental work and developed the required facilities and measurement capabilities. C.H.-G., K.M.D., L.R., J.S.R., M.M.M. and L.P. wrote and prepared the manuscript, to which all authors provided constructive improvements and feedback.

\section{Competing interests}

M.M.M. and H.C.K. have a interest in KMLabs. The other authors declare no competing interests.

\section{Additional information}

Supplementary information is available for this paper at https://doi.org/10.1038/ s41566-018-0304-3.

Reprints and permissions information is available at www.nature.com/reprints. Correspondence and requests for materials should be addressed to K.M.D. or C.H.

Publisher's note: Springer Nature remains neutral with regard to jurisdictional claims in published maps and institutional affiliations.

(C) The Author(s), under exclusive licence to Springer Nature Limited 2018 


\section{Methods}

Experimental generation of SAM-OAM high-harmonic vortices. To generate EUV SAM-OAM vortex beams, we utilized a modified version of the collinear bichromatic scheme typically used in bicircular HHG. The full output of a regenerative Ti:sapphire amplifier $(785 \mathrm{~nm}, 9 \mathrm{~mJ}, 1 \mathrm{kHz}, \mathrm{KMLabs}$ Wyvern HE) is passed into a Mach-Zhender-type interferometer with a branching ratio of $70 / 30$. The more intense of the two arms contains a $200-\mu \mathrm{m}$-thick beta-barium borate crystal, which generates the second harmonic field at $\sim 392 \mathrm{~nm}$. Achromatic waveplates $(\lambda / 2$ and $\lambda / 4)$ are placed in each arm to independently control the polarization of each component, resulting in LCP $\left(785 \mathrm{~nm}, \omega_{1}, \sigma_{1}=-1\right)$ and RCP ( $392 \mathrm{~nm}, \omega_{2}, \sigma_{2}=+1$ ) beams. A multifaceted spiral phase plate (SPP, HoloOr, 16 -steps per phase wrap) is placed in each arm of the interferometer to control the topological charge of the visible vortex wavefront, and different SPPs and their orientations are utilized to generate vortex beams with charges of $\ell_{1}= \pm 1$, \pm 2 and $\ell_{2}= \pm 1$. The purity of the vortex mode and the relative sign of the OAM of each beam is characterized by a combination of a beam profiling camera and a cylindrical lens (see Supplementary Section 1). The two arms are then recombined via a dichroic mirror and spatiotemporal overlap is achieved via a delay stage and independent convex lenses in each arm; generating a bicircular, vortex driving beam. In the experiment, and also in the theoretical simulations (see below), the input beam parameters are carefully adjusted so that the ring of maximum intensity of each driver is spatially overlapped just before the gas jet (see Supplementary Section 1). The combined focus of the bicircular driver is placed just before the exit of a supersonic expansion of Ar gas to drive the HHG process. The generated high harmonics are collected via a 1D+1D (spatio-spectral) spectrometer consisting of a gold-coated cylindrical mirror and a flat grating. The resulting spectrum is recorded with an EUV CCD array (Andor, Newton) at either the flat-field focal plane of the spectrometer (spectral measurements), or at a far field, $\sim 8 \mathrm{~cm}$ beyond this focal plane (EUV vortex profile measurements), which measure both the spectrum and spatial mode of the SAM-OAM harmonics, respectfully.

EUV MCD measurements of SAM-OAM HHG ellipticity. One of the most straightforward ways to determine the helicity of EUV radiation is by exploiting the dichroic absorption of different helicities of light in a suitable chiral material. In this work, we employed EUV MCD measurements to quantify the relative helicity of high harmonics in our SAM-OAM HHG spectra. In EUV MCD, the transmission of RCP and LCP light is slightly different depending on the magnetic moment of a uniformly magnetized film, and the relative (and sometimes absolute) degree of polarization can be determined by measuring the absorption spectrum under different macroscopic magnetizations of the thin magnetic film. In this work, a 20 -nm-thick $\mathrm{Fe}_{0.75} \mathrm{Co}_{0.25}$ alloy film (capped with a double bilayer consisting of $3 \mathrm{~nm}$ each of $\mathrm{Cu} / \mathrm{Ta}$ ) was deposited onto a $200 \mathrm{~nm} \mathrm{Al}$ metal foil and served as the magnetic sample for the EUV MCD measurements. The MCD asymmetry was measured by applying a moderate magnetic field $(\sim 15 \mathrm{mT})$ to the film from an external electromagnet. The film was then oriented at $45^{\circ}$ with respect to the SAM-OAM beam propagation direction to maximize the MCD effect in this geometry. For the spectrally dispersed measurements in Fig. 2, the absorption spectrum of the $\mathrm{Fe}_{0.75} \mathrm{Co}_{0.25}$ film was recorded for $10 \mathrm{~s}$, then the magnetization of the film was switched via the electromagnet, and another absorption spectrum was recorded for another $10 \mathrm{~s}$. This procedure was repeated for 90 paired measurements, giving a total exposure time of $30 \mathrm{~min}$. For the spatially resolved EUV MCD measurements, a total of 120 absorption spectra were taken for each magnetization direction, yielding a total exposure time of $40 \mathrm{~min}$.

Full quantum SAM-OAM HHG simulations including propagation. To simulate bicircular SAM-OAM HHG, we employed a theoretical method that computes both the full quantum single-atom HHG response and subsequent propagation $^{38}$. The propagation is based on the electromagnetic field propagator, in which we discretize the target (gas jet) into elementary radiators ${ }^{38}$. The dipole acceleration of each elementary source is computed using the full quantum strong field approximation, instead of solving directly the time-dependent Schrödinger equation, yielding a performance gain in computational time when computing HHG over the entire target ${ }^{38}$. We assume that the harmonic radiation propagates with the vacuum phase velocity, which is a reasonable assumption for high-order harmonics. Propagation effects in the fundamental field, such as the production of free charges, the refractive index of the neutrals, the group velocity walk-off, as well as absorption in the propagation of the harmonics, are taken into account. Note that although we account for the time-dependent nonlinear phase shifts in the driving fields, nonlinear spatial effects are not taken into account. The spatial structure of the vortex beams considered in our simulations is represented as a Laguerre-Gaussian beam propagating in the $z$ direction (see Supplementary Section 1 for further details). The laser pulses are modelled with a trapezoidal envelope with two cycles of linear turn-on, six cycles of constant amplitude-16 fs-and two cycles of linear turn-off (in cycles of the $\omega_{1}$ field, of $800 \mathrm{~nm}$ in wavelength). The amplitudes $\left(E_{0}\right)$ of the two fields $-\omega_{1}$ of $800 \mathrm{~nm}$ and $\omega_{2}$ of $400 \mathrm{~nm}$ in wavelength -are chosen to obtain the same peak intensity at focus for each driver at the radii of maximum superposition (that is, the brightest intensity rings overlap spatially), yielding a total intensity of $2 \times 10^{14} \mathrm{~W} \mathrm{~cm}^{-2}$. The drivingbeam waists are chosen to overlap at the focal plane and the beam waists for the different cases $\ell= \pm 1, w_{0}=30 \mu \mathrm{m}$, for $\ell= \pm 2, w_{0}=21.4 \mu \mathrm{m}$ and for $\ell= \pm 4, w_{0}=15$ $\mu \mathrm{m}$ (see Supplementary Section 4 ). The gas jet, flowing along the perpendicular direction to the beam propagation is modelled as a Gaussian distribution of $10 \mu$ $\mathrm{m}$ at full width half maximum, and with a peak pressure of $667 \mathrm{~Pa}$ (5 torr). The low thickness of the gas jet is due to computational time limitations; however, based on our previous results of OAM $\mathrm{HHG}^{39}$, we do not foresee any fundamental deviation when considering thicker gas jets closer to the experimental jet employed in this work (a diameter of $150 \mu \mathrm{m}$ ).

\section{Data availability}

The datasets and analysis routines utilized to prepare the data presented in this manuscript are available, free of charge, from the corresponding authors under reasonable request. 